\title{
Capsule Commentary on Oslin et al., A Randomized Clinical Trial of Alcohol Care Management Delivered in Department of Veterans Affairs Primary Care Clinics Versus Specialty Addiction Treatment
}

\author{
Carol A. Malte, MSW \\ Center of Excellence in Substance Abuse Treatment and Education, VA Puget Sound Health Care System, Seattle, WA, USA.
}

$\mathrm{J}$ Gen Intern Med 29(1):184

DOI: $10.1007 / \mathrm{s} 11606-013-2656-1$

(c) Society of General Internal Medicine 2013

\begin{abstract}
A lcohol use disorders (AUDs) are highly prevalent conditions that largely go untreated. Because of this unmet treatment need, the question of whether and for whom alcohol treatment can be successfully delivered in primary care is important. While AUD screening and provision of brief intervention and/or treatment referral as appropriate is encouraged in primary care settings, brief intervention may not be efficacious for patients with heavy alcohol use or dependence, ${ }^{1}$ and referrals often have low rates of acceptance. This trial by Oslin and colleagues ${ }^{2}$ suggests that providing more intensive interventions in primary care settings is an alternative to brief intervention and referral. Patients $(n=163)$ with alcohol dependence were randomized to receive primary care-based Alcohol Care Management (ACM) vs. outpatient addiction specialty care (SC) at three Veterans Affairs (VA) medical centers. Patients randomized to ACM, which consisted of once weekly psychosocial support and encouraged use of oral naltrexone, were much more likely to be engaged in treatment $(\mathrm{OR}=5.36,95 \% \mathrm{CI}=2.99,9.59)$ and much less likely to drink heavily $(\mathrm{OR}=2.16,95 \% \mathrm{CI}=1.27,3.66)$ over a 26-weeks course relative to those randomized to SC. Overall abstinence did not differ between conditions.

Notably, $66 \%$ of ACM patients received prescriptions for naltrexone, compared to $12 \%$ in SC. Despite the fact that use of pharmacotherapy to treat AUDs is strongly recommended by many treatment guidelines, it is underutilized in $\mathrm{VA}^{3}$ and in the general population. ${ }^{4}$ For primary care providers and administrators, ACM may provide a strategy to increase use of AUD pharmacotherapy.

For whom ACM is an appropriate alternative to SC remains to be examined. This trial included patients with depression and anxiety disorders, but it is not known
\end{abstract}

Published online October 16, 2013 whether those with more severe symptomatology benefited from ACM. Likewise, outcomes of those who drank most heavily at baseline should be examined. Implementation of screening and brief intervention in primary care has been hindered by competing provider demands and limited resources, training and support. ${ }^{5}$ Likely, ACM will face similar if not greater challenges. Further study is needed to determine barriers and facilitators to delivering ACM both within and outside of VA.

Conflict of Interest: The author declares no conflicts of interest with this article.

Disclaimer: The views expressed in this article are those of the author and do not necessarily reflect the position or policy of the Department of Veterans Affairs.

Corresponding Author: Carol A. Malte, MSW; Center of Excellence in Substance Abuse Treatment and Education, VA Puget Sound Health Care System, Seattle, WA 98108, USA (e-mail: Carolmalte@va.gov).

\section{REFERENCES}

1. Saitz R. Alcohol screening and brief intervention in primary care: absence of evidence for efficacy in people with dependence or very heavy drinking. Drug Alcohol Rev. 2010;29(6):631-640.

2. Oslin DW, Lynch KG, Maisto SA, Lantinga LJ, McKay JR, Possemato $\mathbf{K}$, Ingram E, Wierzbicki M. A randomized clinical trial of alcohol care management delivered in department of veterans affairs primary care clinics versus specialty addiction treatment. J Gen Intern Med. 2013; doi: 10.1007/s11606-013-2625-8.

3. Harris AHS, Kivlahan DR, Bowe T, et al. Pharmacotherapy of alcohol use disorders in the Veterans Health Administration. Psychiatr Serv. 2010;61(4):392-8.

4. Mark TL, Kassed CA, Vandivort-Warren R, Levit KR, Kranzler HR. Alcohol and opioid dependence medications: prescription trends, overall and by physician specialty. Drug Alcohol Depend. 2009;99(1-3):345-349.

5. Johnson M, Jackson R, Guillaume L, Meier P, Goyder E. Barriers and facilitators to implementing screening and brief intervention for alcohol misuse: a systematic review of qualitative evidence. J Public Health. 2011;33(3):412-21. 